\title{
Coffee, tea and caffeine intake and the risk of severe depression in middle-aged Finnish men: the Kuopio Ischaemic Heart Disease Risk Factor Study
}

\author{
Anu Ruusunen ${ }^{1, *}$, Soili M Lehto ${ }^{2,3}$, Tommi Tolmunen², Jaakko Mursu', \\ George A Kaplan ${ }^{4}$ and Sari Voutilainen ${ }^{1}$ \\ ${ }^{1}$ Institute of Public Health and Clinical Nutrition, Kuopio Campus, University of Eastern Finland, PO Box 1627, \\ 70211 Kuopio, Finland: ${ }^{2}$ Department of Psychiatry, Kuopio University Hospital and University of Eastern Finland, \\ Kuopio, Finland: ${ }^{3}$ Kuopio Psychiatric Center, Kuopio City and Kuopio University Hospital, Kuopio, Finland: \\ ${ }^{4}$ Center for Social Epidemiology and Population Health, The University of Michigan School of Public Health, \\ Ann Arbor, MI, USA
}

Submitted 27 March 2009: Accepted 10 February 2010: First published online 1 April 2010

\begin{abstract}
Objective: Only a few cross-sectional studies have assessed the association between coffee, tea and caffeine and the risk of depression. Our aim was to determine the association in a population-based cohort study.

Design: The population-based Kuopio Ischaemic Heart Disease Risk Factor Study cohort was recruited between 1984 and 1989 and followed until the end of 2006. We investigated the association between the intake of coffee, tea and caffeine and depression.

Setting: Eastern Finland.

Subjects: Middle-aged men ( $n$ 2232).

Results: Altogether, forty-nine men received a discharge diagnosis of depression. We classified subjects into quartiles according to their mean daily coffee intake: non-drinkers ( $n$ 82), light drinkers $(<375 \mathrm{ml} / \mathrm{d}, n$ 517), moderate drinkers $(375-813 \mathrm{ml} / \mathrm{d}, n$ 1243) and heavy drinkers $(>813 \mathrm{ml} / \mathrm{d}, n 390)$. Heavy drinkers had a decreased risk $(\mathrm{RR}=0 \cdot 28,95 \% \mathrm{CI} 0 \cdot 08,0 \cdot 98)$ for depression when compared with non-drinkers, after adjustment for age and examination years. Further adjustment for socio-economic status, alcohol consumption, smoking, maximal oxygen uptake, BMI and the energy-adjusted daily intakes of folate and PUFA did not attenuate this association (relative risk $(\mathrm{RR})=0 \cdot 23,95 \% \mathrm{CI} 0 \cdot 06,0 \cdot 83$ ). No associations were observed between depression and intake of tea (drinkers $v$. non-drinkers; RR $=1 \cdot 19,95 \%$ CI $0 \cdot 54,2 \cdot 23$ ) or caffeine (highest quartile $v$. lowest quartile; $\mathrm{RR}=0 \cdot 99,95 \% \mathrm{CI} 0 \cdot 40,2 \cdot 45)$.

Conclusions: Coffee consumption may decrease the risk of depression, whereas no association was found for tea and caffeine intake.
\end{abstract}

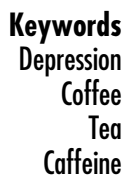

Coffee and tea are two of the most commonly consumed beverages in the world, and the health effects of these drinks have evoked wide interest. However, there is still only little information about their effect on morbidity ${ }^{(1-3)}$. Moreover, it is unclear whether coffee and tea drinking or caffeine intake is associated with depression, a major condition affecting public health.

Caffeine is one of the most widely used psychoactive substances, and may contribute to many psychiatric problems, such as depression, anxiety and psychosis ${ }^{(4)}$. Coffee is the primary source of caffeine all over the world $^{(5)}$. However, only a few studies have been published on the association between coffee and tea consumption or caffeine intake and depression ${ }^{(6-8)}$. Moreover, the health effects of regular caffeine intake have been poorly studied, although the acute or short-term effects are well known. Earlier, tea consumption has been found to be associated with decreased risk of being depressed $^{(9)}$. An inverse relationship between tea drinking and the risk of being depressed was found in a general population sample in a cross-sectional setting ${ }^{(9)}$. However, caffeine use has been reported to increase depressive symptoms in cross-sectional studies ${ }^{(6,7,10)}$. A review of effects of caffeine reported that Beck Depression Inventory Scale scores increased proportionally with increased caffeine intake in psychiatric inpatients ${ }^{(11)}$. In contrast, in a recent study caffeine intake was found to be associated with decreased risk of clinical depression ${ }^{(8)}$. 
Separate analyses were conducted on caffeine intake derived from coffee and tea and revealed similar effects on clinical depression, which suggest that it is caffeine rather than any other compound in caffeine-containing drinks that may produce the protective effect on depression $^{(8)}$. However, it has been assumed that in the long term, caffeine has only little effect on mood ${ }^{(12)}$. Furthermore, since the causality cannot be judged from the cross-sectional studies, the possibility of coffee use as a self-medication to relieve the symptoms of depression cannot be excluded in these studies.

Existing literature on the connection between depression and the intake of coffee, tea or caffeine is scarce, and prospective studies are needed to clarify the association. Therefore, we examined whether coffee or tea consumption or caffeine intake is associated with the risk of depression in middle-aged Finnish men.

\section{Materials and methods}

\section{Study population}

The Kuopio Ischaemic Heart Disease Risk Factor Study (KIHD) is an ongoing population-based cohort study designed to investigate risk factors for CVD and other chronic diseases in middle-aged men from Eastern Finland $^{(13)}$. A total of 2682 participants $(82.9 \%$ of those eligible), aged 42, 48, 54 and 60 years, were enrolled at baseline between 1984 and 1989. The participants gave written informed consent. The study population ( $n$ 2232) included those men who were not found to be depressive at the baseline examinations and had no previously diagnosed psychiatric disorder. The study protocol was approved by the research ethics committee of the University of Kuopio and Kuopio University Hospital.

\section{Exclusion criteria and outcomes}

Depressive symptoms were assessed with the eighteen-item Human Population Laboratory (HPL) Depression Scale ${ }^{(14)}$ at baseline. The scale consists of items dealing with mood disturbance, negative self-concept, loss of energy, problems with eating and sleeping, trouble with concentration and psychomotor retardation or agitation. The HPL Depression Score was developed especially for screening general population samples ${ }^{(14)}$, and it also conceptually resembles other brief symptom checklists such as the Center for Epidemiological Studies Depression Scale (CES-D) ${ }^{(15,16)}$. The HPL depression score is generated by assessing one point for each true or false answer that is indicative of depression. A cut-off score of five or more has earlier been used to define depression ${ }^{(14,16,17)}$. Those who scored five or more at baseline ( $n 271,10.9 \%$ of participants) were considered depressed, and were thus excluded from the analysis.

We used severe depression diagnosed by a physician as an outcome. Data included participants who received a discharge diagnosis of depressive disorder during the follow-up until the end of December 2006. The information was obtained by a computer linkage to the national hospital discharge register. The participants who were hospitalised and had been diagnosed as having major depression (International Classification of Diseases (ICD)-9: 2961-, ICD-10: F32.1-3, F33.1-3; $n$ 28), depression, an otherwise unspecified disorder (ICD-9: 2968A, ICD-10: F32.9, F33.9; $n$ 14), chronic depression (ICD-8: 300.41, ICD-9: 3004A, ICD-10: F34.1; $n$ 4) or adjustment disorder with depressive symptoms (ICD-9: 3090A; $n$ 3) during the follow-up period were regarded as cases.

\section{Assessment of coffee and tea consumption and otber nutritional factors}

The dietary intake of foods and beverages was quantitatively assessed by a $4 \mathrm{~d}$ food recording at the KIHD study baseline. Participants filled the instructed and nutritionistchecked $4 \mathrm{~d}$ food recording by using conventional household measures, including cups of coffee and tea. Nutrient intakes were calculated using the Finnish Nutrica ${ }^{\circledR}$ software (The Social Insurance Institution of Finland, Turku, Finland), which is mainly compiled using Finnish values for the nutrient composition of foods. Coffee and tea are the main sources of caffeine in our study population, since it was very uncommon to use other sources of caffeine in Finland in the late 1980s. We calculated the caffeine intake of participants from these most important sources of caffeine. In these calculations, we assumed that $100 \mathrm{ml}$ of coffee and tea contain $100 \mathrm{mg}$ and $40 \mathrm{mg}$ of caffeine, respectively. The folate intake was adjusted for dietary energy intake using the regression residual method ${ }^{(18)}$. Energy adjustment is based on the notion that a larger, more physically active person requires a higher energy intake, which is associated with a higher absolute intake of all nutrients.

\section{Otber measurements}

Smoking habits, alcohol consumption, marital status and education at the baseline were assessed using questionnaires as described previously ${ }^{(19,20)}$. Description of the determination of maximal oxygen uptake has also been published previously ${ }^{(19)}$. The variable of adult socio-economic status was formed from a variety of indicators, including current income, current and previous occupations, the highest level of education, the perception of financial security, and housing tenure. The weight and height of the participants were measured by a study nurse, and BMI was calculated as the ratio of weight in kilograms to the square of height in metres $\left(\mathrm{kg} / \mathrm{m}^{2}\right)$.

\section{Statistical methods}

The heterogeneity of the means of baseline variables between those who had received a discharge diagnosis of depression during the follow-up and those who remained free of depression, as well as the differences between coffee drinkers and non-drinkers, was tested by using the ANOVA test, Student's $t$ test, Mann-Whitney U-test, $\chi^{2}$ test 
and Fisher's exact test. The non-parametric Mann-Whitney $U$-test was utilised when the distribution of the values was skewed.

The relative risk (RR) of depression was examined using the Cox proportional hazards model adjusted for age and examination years (Model 1) and for socio-economic status, smoking, alcohol consumption, maximal oxygen uptake, BMI and daily intakes of folate and PUFA (Model 2). Further adjustment for marital status, medical comorbidity (hypertension, diabetes and heart disease), leisuretime activity, energy intake, the energy-adjusted daily intakes of eicosapentaenoic and docosahexaenoic acids, and use of dairy products did not appreciably change the associations (data not shown). We also repeated the analyses substituting the intake of PUFA with MUFA and SFA, which did not attenuate the results (data not shown). Model 3 included covariates in Model 2 plus HPL Depression Scale scores. To investigate the consistency of coffee drinking habits, we examined the correlation of coffee consumption between the KIHD baseline and 11-year follow-up examinations using Pearson's correlation coefficient.

The participants were divided into quartiles according to coffee drinking: non-drinkers, light drinkers $(<375 \mathrm{ml} /$ d), moderate drinkers $(375-813 \mathrm{ml} / \mathrm{d})$ and heavy drinkers $(>813 \mathrm{ml} / \mathrm{d})$. Because of the low amount of tea consumed by the tea drinkers (mean intake: $105 \mathrm{ml} / \mathrm{d}$ ), participants were categorised into tea drinkers and non-drinkers. The cohort was also divided into quartiles based on caffeine intake: $<425 \mathrm{mg} / \mathrm{d}, \quad 425-594 \mathrm{mg} / \mathrm{d}, \quad 595-781 \mathrm{mg} / \mathrm{d}$ and $>781 \mathrm{mg} / \mathrm{d}$. All statistical analyses were conducted using the Statistical Package for the Social Sciences statistical software package version 14.0 for Windows (SPSS Inc., Chicago, IL, USA).

\section{Results}

Altogether, seventy-three participants received a unipolar depressive disorder as a discharge diagnosis during the follow-up period of $17 \cdot 5$ years. After excluding those with significant depressive symptoms at baseline ( $n$ 16) and those with a history of mental illness ( $n$ 8), forty-nine participants with depression remained for final analysis.

At baseline, the men who were hospitalised for depression had higher energy intake and higher HPL depression scores than those who had no severe depression during the follow-up (Table 1). There was a significant correlation between the reported coffee drinking at baseline and the 11-year follow-up $(r=0.54, \quad P<0 \cdot 001)$. The baseline characteristics for the coffee drinkers and non-drinkers are presented in the Table 2 . The coffee drinkers had a lower intake of folate, and were more likely to be smokers than the non-drinkers. A total of $4.9 \%$ of the non-drinkers, $1.3 \%$ of the light drinkers, $2 \cdot 6 \%$ of the moderate drinkers and $1.5 \%$ of the heavy drinkers received a discharge diagnosis of depression during the follow-up.

When comparing heavy drinkers with non-drinkers, they had a decreased risk of severe depression ( $\mathrm{RR}=0 \cdot 28,95 \%$ CI 0.08, 0.98; Table 3, Model 1). Further adjustments (Models 2 and 3) did not significantly attenuate the association. When comparing coffee drinkers ( $n$ 2150) and nondrinkers ( $n$ 82), there was a trend towards decreased risk of depression after adjustment for age and examination years $(\mathrm{RR}=0 \cdot 41,95 \%$ CI $0 \cdot 14,1 \cdot 12, P=0 \cdot 081)$. Further adjustments (Models 2 and 3 ) did not alter the association.

Tea drinking or caffeine intake was not associated with the risk of depression. Table 3 presents the risks of severe depression in tea drinkers $v$. non-drinkers and in quartiles of caffeine intake. The results remained similar when

Table 1 Characteristics of the study population at baseline according to the discharge diagnosis of depression during the $17 \cdot 5$-year follow-up

\begin{tabular}{|c|c|c|c|c|c|c|c|}
\hline & \multicolumn{2}{|c|}{ All $(n$ 2232) } & \multicolumn{2}{|c|}{ Depressed ( $n$ 49) } & \multicolumn{2}{|c|}{ Others $(n 2183)$} & \multirow[b]{2}{*}{$P$ value for difference* } \\
\hline & Mean or \% & SD & Mean or \% & SD & Mean or \% & SD & \\
\hline Coffee consumption (ml/d) & 565 & 293 & 548 & 245 & 565 & 294 & $0 \cdot 68 t$ \\
\hline Tea consumption $(\mathrm{ml} / \mathrm{d})$ & 105 & 183 & 121 & 207 & 105 & 182 & $0.54 t$ \\
\hline Total caffeine intake (mg/d) & 494 & 221 & 487 & 177 & 494 & 221 & $0 \cdot 82+$ \\
\hline Energy intake $(\mathrm{MJ} / \mathrm{d})$ & 9.9 & $2 \cdot 6$ & $11 \cdot 4$ & $2 \cdot 8$ & $9 \cdot 9$ & $2 \cdot 6$ & $<0.001 \dagger$ \\
\hline Energy-adjusted folate intake $(\mu \mathrm{g} / \mathrm{d})$ & 255 & 56 & 241 & 63 & 256 & 56 & $0.07 t$ \\
\hline PUFA intake (E\%) & $4 \cdot 6$ & $1 \cdot 4$ & 4.7 & $1 \cdot 3$ & 4.5 & $1 \cdot 4$ & $0.41+$ \\
\hline Alcohol intake (g/week) & $71 \cdot 8$ & $133 \cdot 6$ & $75 \cdot 3$ & $101 \cdot 3$ & $71 \cdot 7$ & $134 \cdot 2$ & $0 \cdot 85 \ddagger$ \\
\hline Age (years) & $53 \cdot 0$ & $5 \cdot 2$ & $51 \cdot 6$ & $5 \cdot 8$ & $53 \cdot 0$ & $5 \cdot 2$ & $0.07 t$ \\
\hline Marital status: living alone (\%) & 12 & & 16 & & 12 & & $0.24 \S$ \\
\hline Smoking (\%) & 31 & & 33 & & 31 & & $0 \cdot 46 \|$ \\
\hline BMI $\left(\mathrm{kg} / \mathrm{m}^{2}\right)$ & $26 \cdot 8$ & $3 \cdot 5$ & $27 \cdot 1$ & $3 \cdot 6$ & $26 \cdot 8$ & $3 \cdot 5$ & $0.58+$ \\
\hline Maximal oxygen uptake (ml/kg per min) & $30 \cdot 9$ & $7 \cdot 6$ & $32 \cdot 0$ & $6 \cdot 9$ & $30 \cdot 9$ & $7 \cdot 6$ & $0 \cdot 31+$ \\
\hline Socio-economic status (points) & $9 \cdot 18$ & $4 \cdot 6$ & $10 \cdot 18$ & 3.9 & $9 \cdot 16$ & $4 \cdot 6$ & $0 \cdot 12 \dagger$ \\
\hline HPL depression score (points) & $1 \cdot 3$ & $1 \cdot 3$ & 1.9 & 1.5 & $1 \cdot 3$ & $1 \cdot 3$ & $0.002 t$ \\
\hline
\end{tabular}

E\%, percentage of energy; HPL, Human Population Laboratory.

${ }^{*} P$ value for the difference between depressed and others.

tStudent's $t$ test.

$\ddagger$ Mann-Whitney U-test

$\S \chi^{2}$ test.

IIFishers's exact test. 
Table 2 Baseline characteristics of the study population according to coffee consumption

\begin{tabular}{|c|c|c|c|c|c|c|c|c|c|}
\hline & \multicolumn{8}{|c|}{ Coffee intake category ${ }^{*}$} & \multirow[b]{3}{*}{$P$ valuet } \\
\hline & \multicolumn{2}{|c|}{ None $(n$ 82) } & \multicolumn{2}{|c|}{ Light ( $n 517)$} & \multicolumn{2}{|c|}{ Moderate $(n 1243)$} & \multicolumn{2}{|c|}{ Heavy ( $n$ 390) } & \\
\hline & Mean or $\%$ & SD & Mean or \% & SD & Mean or \% & SD & Mean or $\%$ & SD & \\
\hline Coffee consumption (ml/d) & 0 & 0 & 250 & 99 & 595 & 118 & 1007 & 191 & $<0.001 \ddagger$ \\
\hline Tea consumption (ml/d) & 349 & 348 & 180 & 213 & 76 & 145 & 47 & 113 & $<0.001 \ddagger$ \\
\hline Total caffeine intake $(\mathrm{mg} / \mathrm{d})$ & 139 & 115 & 272 & 116 & 506 & 132 & 824 & 181 & $<0.001 \ddagger$ \\
\hline Energy intake $(\mathrm{MJ} / \mathrm{d})$ & $10 \cdot 7$ & 2.9 & $9 \cdot 1$ & $2 \cdot 3$ & 9.9 & $2 \cdot 5$ & $11 \cdot 0$ & $3 \cdot 0$ & $0.01 \ddagger$ \\
\hline Energy-adjusted folate intake $(\mu \mathrm{g} / \mathrm{d})$ & 275 & 75 & 264 & 55 & 254 & 55 & 244 & 54 & $0.001 \ddagger$ \\
\hline PUFA intake (E\%) & 4.5 & $1 \cdot 4$ & $4 \cdot 7$ & $1 \cdot 4$ & $4 \cdot 6$ & $1 \cdot 4$ & $4 \cdot 3$ & $1 \cdot 3$ & $0.61 \ddagger$ \\
\hline Alcohol consumption (g/week) & 70 & 145 & 78 & 131 & 71 & 138 & 65 & 108 & $0.92 \S$ \\
\hline Age (years) & $52 \cdot 6$ & $5 \cdot 0$ & $53 \cdot 3$ & $5 \cdot 2$ & $53 \cdot 0$ & $5 \cdot 2$ & $52 \cdot 3$ & $5 \cdot 2$ & $0.56 \ddagger$ \\
\hline Marital status: living alone (\%) & $8 \cdot 6$ & & $10 \cdot 5$ & & $11 \cdot 4$ & & $16 \cdot 9$ & & $0.05 \|$ \\
\hline Smoking (\%) & 11 & & 23 & & 30 & & 50 & & $<0.001$ \\
\hline BMI $\left(\mathrm{kg} / \mathrm{m}^{2}\right)$ & $26 \cdot 2$ & $3 \cdot 3$ & $27 \cdot 0$ & $3 \cdot 8$ & $26 \cdot 9$ & $3 \cdot 4$ & $26 \cdot 4$ & $3 \cdot 2$ & $0 \cdot 12 \ddagger$ \\
\hline Maximal oxygen uptake (ml/kg per min) & $31 \cdot 9$ & $6 \cdot 7$ & $30 \cdot 2$ & $7 \cdot 5$ & $31 \cdot 1$ & $7 \cdot 5$ & $31 \cdot 2$ & $8 \cdot 2$ & $0.06 t$ \\
\hline Socio-economic status (points) & $9 \cdot 7$ & $4 \cdot 7$ & 8.5 & $4 \cdot 7$ & $9 \cdot 1$ & $4 \cdot 6$ & $10 \cdot 4$ & 4.5 & $<0.001 t$ \\
\hline HPL depression score (points) & $1 \cdot 25$ & $1 \cdot 29$ & $1 \cdot 34$ & $1 \cdot 31$ & $1 \cdot 32$ & $1 \cdot 27$ & $1 \cdot 22$ & $1 \cdot 23$ & $0.64 \|$ \\
\hline
\end{tabular}

E\%, percentage of energy; HPL, Human Population Laboratory.

*Light: <375 ml/d; moderate: $375-813 \mathrm{ml} / \mathrm{d}$; heavy: $>813 \mathrm{ml} / \mathrm{d}$.

$+P$ value for the difference between coffee drinkers and non-drinkers.

$\ddagger$ Student's $t$ test.

$\S$ Mann-Whitney U-test.

$\| \chi^{2}$ test.

-Fishers's exact test.

Table 3 Relative risk (RR) of severe depression during the follow-up of $17 \cdot 5$ years in middle-aged men according to daily consumption of coffee or tea or intake of caffeine

Coffee intake category

\begin{tabular}{|c|c|c|c|c|c|c|c|c|c|}
\hline & \multicolumn{2}{|c|}{ None $(n$ 82) } & \multicolumn{2}{|c|}{ Light ( $n$ 517) } & \multicolumn{2}{|c|}{ Moderate $(n 1243)$} & \multicolumn{2}{|c|}{ Heavy (n 390) } & \\
\hline & $n$ & $\%$ & $n$ & $\%$ & $n$ & $\%$ & $n$ & $\%$ & \\
\hline \multirow[t]{2}{*}{ Events } & 4 & 4.9 & 7 & $1 \cdot 3$ & 32 & $2 \cdot 6$ & 6 & $1 \cdot 5$ & \\
\hline & $n$ & & $\mathrm{RR}$ & $95 \% \mathrm{Cl}$ & $\mathrm{RR}$ & $95 \% \mathrm{Cl}$ & $\mathrm{RR}$ & $95 \% \mathrm{Cl}$ & $P$ value \\
\hline $\begin{array}{l}\text { Model } 1 \ddagger \\
\text { Model } 2 \S\end{array}$ & $\begin{array}{l}1 \\
1\end{array}$ & & $\begin{array}{l}0.27 \\
0.28\end{array}$ & $\begin{array}{l}0.08,0.93 \\
0.08,0.96\end{array}$ & $\begin{array}{l}0.49 \\
0.45\end{array}$ & $\begin{array}{l}0 \cdot 17,1 \cdot 40 \\
0 \cdot 16,1 \cdot 29\end{array}$ & $\begin{array}{l}0.28 \\
0.23\end{array}$ & $\begin{array}{l}0.08,0.98 \\
0.06,0.83\end{array}$ & $\begin{array}{l}0.047 \\
0.025\end{array}$ \\
\hline Model 3॥ & 1 & & 0.29 & $0.08,0.98$ & 0.48 & $0 \cdot 17,1 \cdot 36$ & 0.25 & $0.07,0.91$ & 0.035 \\
\hline
\end{tabular}

Tea consumption category

\begin{tabular}{|c|c|c|c|c|c|c|c|c|c|}
\hline & \multicolumn{2}{|c|}{ Non-tea drinker ( $n$ 1264) } & \multicolumn{2}{|c|}{ Tea drinker ( $n$ 968) } & & & & & \\
\hline & $n$ & $\%$ & $n$ & $\%$ & & & & & \\
\hline \multirow[t]{2}{*}{ Events (\%) } & 25 & $1 \cdot 9$ & 24 & $2 \cdot 5$ & & & & & \\
\hline & $n$ & & $\mathrm{RR}$ & $95 \% \mathrm{Cl}$ & & & & & $P$ value \\
\hline \multirow{6}{*}{$\begin{array}{l}\text { Model } 1 \ddagger \\
\text { Model } 2 \S \\
\text { Model } 3 \|\end{array}$} & 1 & & $1 \cdot 19$ & $0 \cdot 69,2 \cdot 08$ & & & & & 0.549 \\
\hline & 1 & & 1.43 & $0 \cdot 80,2 \cdot 56$ & & & & & 0.222 \\
\hline & 1 & & $1 \cdot 40$ & $0 \cdot 78,2 \cdot 51$ & & & & & $0 \cdot 252$ \\
\hline & \multicolumn{9}{|c|}{ Quartiles of caffeine intake } \\
\hline & \multicolumn{2}{|c|}{$<425 \mathrm{mg} / \mathrm{d}$} & \multicolumn{2}{|c|}{$425-594 \mathrm{mg} / \mathrm{d}$} & \multicolumn{2}{|c|}{$595-781 \mathrm{mg} / \mathrm{d}$} & \multicolumn{2}{|c|}{$>781 \mathrm{mg} / \mathrm{d}$} & \\
\hline & $n$ & $\%$ & $n$ & $\%$ & $n$ & $\%$ & $n$ & $\%$ & \\
\hline \multirow[t]{2}{*}{ Events } & 9 & $1 \cdot 6$ & 12 & $2 \cdot 1$ & 18 & $3 \cdot 3$ & 10 & $1 \cdot 8$ & \\
\hline & $n$ & & $\mathrm{RR}$ & $95 \% \mathrm{Cl}$ & $\mathrm{RR}$ & $95 \% \mathrm{Cl}$ & RR & $95 \% \mathrm{Cl}$ & $P$ value \\
\hline Model 1‡ & 1 & & $1 \cdot 22$ & $0.52,2 \cdot 91$ & 1.97 & $0 \cdot 89,4 \cdot 42$ & 0.99 & $0 \cdot 40,2 \cdot 45$ & 0.981 \\
\hline Model $2 \S$ & 1 & & $1 \cdot 12$ & $0.47,2 \cdot 67$ & $1 \cdot 83$ & $0 \cdot 82,4 \cdot 11$ & 0.85 & $0 \cdot 34,2 \cdot 16$ & 0.739 \\
\hline Model 3\| & 1 & & 1.07 & $0.45,2.56$ & $1 \cdot 83$ & $0.82,4.09$ & 0.85 & $0 \cdot 34,2 \cdot 15$ & $0 \cdot 732$ \\
\hline
\end{tabular}

$\mathrm{RR}$ is derived from Cox proportional hazards models.

*Light: <375 ml/d; moderate: $375-813 \mathrm{ml} / \mathrm{d}$; heavy: $>813 \mathrm{ml} / \mathrm{d}$.

t $P$ value for the difference between heavy drinkers and non-drinkers.

$\ddagger$ Adjusted for age and examination years.

§Adjusted for Model 1 and socio-economic status, smoking, alcohol consumption, maximal oxygen uptake, BMI, daily intake of folate and PUFA.

$\|$ Adjusted for Model 2 and Human Population Laboratory Depression Scale scores.

$\uparrow P$ value for the difference between the lowest and the highest caffeine intake groups. 
caffeine intake groups were categorised as medians (RR $=1.31 ; 95 \%$ CI $0.74,2.31 ; P=0.355)$ or tertiles (highest tertile $v$. lowest tertile; RR $=1 \cdot 14 ; 95 \%$ CI $0 \cdot 53$, 2.44; $P=0 \cdot 744)$.

\section{Discussion}

To our knowledge, this is the first prospective follow-up study examining the associations between the intake of coffee, tea and caffeine and depression. Our main finding was that coffee drinking may be associated with lower risk of severe depression. In contrast, tea drinking or intake of caffeine was not associated with the risk of depression.

The main strength of our study is a prospective followup setting. Prospective studies give more reliable information on the causal relationship than cross-sectional studies. The large-scale control of confounding factors was also possible due to the numerous measurements carried out at the baseline. Another strength is reliable nutrient data collection. The food records were collected over four consecutive days. Coffee consumption usually remains relatively constant from day to day, and thus a food record can be considered a reliable method for its assessment. A qualified nutritionist provided the necessary instructions, and checked completed food records with the participants, which added validity.

Our study also has some limitations. First, despite the relatively large sample size, the number of cases was relatively low. Because we have no national register of depressed outpatients in Finland, the study was limited to participants with severe depression requiring hospitalisation. Although we observed a significant association between coffee drinking and the risk of severe depression, it is possible that the low number of cases affected our results. Changes in coffee drinking habits during the long follow-up period could also undermine the observed results of our study. However, habits remained relatively stable in our study population $(r=0.54, P<0.001)$.

Recent epidemiological and experimental studies have shown positive effects of coffee and tea drinking on various aspects of health ${ }^{(1,2,8)}$, but the information related to depression is unclear. Cross-sectional studies on caffeine intake and depressive symptoms have reported inconsistent findings ${ }^{(6,7,10)}$. Interestingly, coffee consumption has been suggested to be beneficial in neurodegenerative diseases in which depressive mood is a common symptom $^{(3,21,22)}$. It has been suggested that the possible protective effect of caffeine-containing drinks on depression as well as neurodegenerative diseases might be especially due to caffeine ${ }^{(3,8)}$. Caffeine could mediate the protective effect of coffee or tea by stimulating the central nervous system ${ }^{(23)}$ and enhancing dopaminergic neurotransmission ${ }^{(24)}$. However, our results are not in a line with the hypothesis that the inverse association between coffee drinking and depression is mediated by caffeine.
We have shown earlier in the same KIHD cohort that low dietary intake of folate was associated with the increased risk of depression $^{(17)}$. This has also been shown in other prospective studies ${ }^{(25,26)}$. Although the coffee drinkers had a lower intake of folate than the non-drinkers (Table 2), adjustments for folate and other potential confounders did not attenuate the association found between coffee drinking and the risk of depression. In addition, coffee intake is usually associated with other unhealthy lifestyle factors such as smoking ${ }^{(27)}$, low physical activity, alcohol consumption and high fat intake ${ }^{(28)}$. In our study population, coffee drinkers had lower socioeconomic status and were more often smokers (Table 2). However, they had decreased risk of depression. Thus, our findings suggest that coffee drinking may act as an independent protective factor from depression.

In a previous Finnish study it was observed that there was an inverse association between daily tea drinking and the risk of being depressed ${ }^{(9)}$. In our cohort, $43 \%$ of participants were tea drinkers, but the mean intake of tea was relatively low, $105 \mathrm{ml} / \mathrm{d}$, and $52 \%$ of the tea drinkers drank $<200 \mathrm{ml}$ of tea daily. However, in the other Finnish study tea drinking was even more unusual (22\% of participants) and $76 \%$ of tea drinkers consumed tea 1-2 cups/d ${ }^{(9)}$. Regardless of the relatively low amounts of tea consumed, tea drinkers had a significantly lower prevalence of depression in that study. Coffee drinking was slightly more common (96\%) in our study than in the other Finnish study $(82 \%)^{(9)}$. In contrast to our results, in the previous Finnish study, coffee drinking had no association with depression. Therefore, both of these studies reinforce the hypothesis that the inverse association between tea or coffee drinking and depression is due to other biologically active compounds than caffeine ${ }^{(9)}$.

The mechanisms behind the possible positive effects of coffee consumption on depression require further investigation. In addition to caffeine, coffee contains many other functional components such as chlorogenic acid and ferulic acid $^{(3)}$. Coffee is also rich in other biologically active substances, such as nicotinic acid, trigonelline, quinolinic acid, tannic acid and pyrogallic acid ${ }^{(29)}$. Interestingly, chlorogenic acid has been suggested to have anti-inflammatory effects in vivo ${ }^{(30)}$. Since depression is considered to be characterised by low-grade inflammation ${ }^{(31,32)}$, the anti-inflammatory effects of coffee, especially in the heavy coffee drinker group, might at least partly explain our observations. Furthermore, oxidation may play an important role in depression ${ }^{(33)}$, and the phenolic acids of coffee, especially chlorogenic acid and caffeic acid, have been found to have antioxidant effects both in vitro ${ }^{(34)}$ and in vivo ${ }^{(35)}$.

\section{Conclusion}

Coffee consumption, but not tea consumption or caffeine intake, may be associated with the decreased risk of 
severe depression in middle-aged or older Finnish men. Further studies are required to investigate the mechanisms behind these observations.

\section{Acknowledgements}

The present study was supported by the Finnish Graduate School of Psychiatry, the Juho Vainio Foundation and the Yrjö Jahnsson Foundation. None of the authors had any personal or financial conflict of interest. The contributions of each author are as follows; A.R. contributed to the data collection, performed statistical analyses and wrote the manuscript by incorporating critical inputs from all authors. S.V. and G.A.K. conducted the study and contributed to the data collection and G.A.K. contributed to the study design. S.M.L., T.T., J.M. and S.V. critically revised the manuscript for important intellectual content and provided statistical expertise. All authors have seen and had the opportunity to comment on the final manuscript. The authors especially thank the previous and present personnel and administration of the Research Institute of Public Health, University of Kuopio, without whom this study could not have been completed.

\section{References}

1. Binns CW, Lee AH \& Fraser ML (2008) Tea or coffee? A case study on evidence for dietary advice. Public Health Nutr 11, 1132-1141.

2. Higdon JV \& Frei B (2006) Coffee and health: a review of recent human research. Crit Rev Food Sci Nutr 46, 101-123.

3. Dòrea JG \& da Costa TH (2005) Is coffee a functional food? BrJ Nutr 93, 773-782.

4. Broderick P \& Benjamin AB (2004) Caffeine and psychiatric symptoms: a review. J Okla State Med Assoc 97, 538-542.

5. Nawrot P, Jordan S, Eastwood J et al. (2003) Effects of caffeine on human health. Food Addit Contam 20, 1-30.

6. Greden JF, Fontaine P, Lubetsky M et al. (1978) Anxiety and depression associated with caffenism among psychiatric in-patients. Am J Psychiatry 135, 963-966.

7. Gilliland K \& Andrews D (1981) Ad lib caffeine consumption, symptoms of caffenism and academic performance. Am J Psychiatry 138, 512-514.

8. Smith AP (2009) Caffeine, cognitive failures and health in a non-working community sample. Hum Psychopharmacol Clin Exp 24, 29-34.

9. Hintikka J, Tolmunen T, Honkalampi K et al. (2005) Daily tea drinking is associated with a low level of depressive symptoms in the Finnish general population. Eur $J$ Epidemiol 20, 359-363.

10. James JE \& Crosbie J (1987) Somatic and psychological health implications of heavy caffeine use. BrJ Addict $\mathbf{8 2}$, 503-509.

11. Clementz GL \& Dailey JW (1988) Psychotropic effects of caffeine. Am Fam Physician 37, 167-172.

12. James JE (1991) Psychopharmacology. In Caffeine and Health, pp. 280-300 [JE James, editor]. London: Academic Press.

13. Salonen JT (1988) Is there a continuing need for longitudinal epidemiologic research? The Kuopio Ischaemic Heart Disease Risk Factor Study. Ann Clin Res 20, 46-50.
14. Kaplan GA, Roberts RE, Camacho TC et al. (1987) Psychosocial predictors of depression: prospective evidence from the Human Population Laboratory Studies. Am J Epidemiol 125, 206-220.

15. Roberts RE (1981) Prevalence of depressive symptoms among Mexican Americans. J Nerv Ment Dis 169, 213-219.

16. Roberts RE \& O' Keefe SJ (1981) Sex differences in depression re-examined. J Health Soc Behav 22, 394-400.

17. Tolmunen T, Hintikka J, Ruusunen A et al. (2004) Dietary folate and the risk of depression in Finnish middle-aged men. A prospective follow-up study. Psychother Psychosom 73, 334-339.

18. Willett W \& Stampfer M (1998) Implications of total energy intake for epidemiologic analyses. In Nutritional Epidemiology, pp. 288-298 [W Willett, editor]. New York, NY: Oxford University Press.

19. Salonen JT, Nyyssönen K, Korpela H et al. (1992) High stored iron levels are associated with excess risk of myocardial infarction in Eastern Finnish men. Circulation 86, 803-811.

20. Lynch J, Helmrich SP, Lakka TA et al. (1996) Moderately intense physical activities and high levels of cardiorespiratory fitness reduce the risk of non-insulin-dependent diabetes mellitus in middle-aged men. Arch Intern Med 156, 1307-1314.

21. Hernan MA, Takkouche B, Caamano-Isorna F et al. (2002) A meta-analysis of coffee drinking, cigarette smoking and the risk of Parkinson's disease. Ann Neurol 52, 276-284.

22. Eskelinen MH, Ngandu T, Tuomilehto J et al. (2009) Midlife coffee and tea drinking and the risk of late-life dementia: a population-based CAIDE study. J Alzheimers Dis 16, 85-91.

23. Szekely T (1997) Caffeine as a stimulant against suicide. Arch Intern Med 157, 243-244.

24. Kaasinen V, Aalto S, Nagren K et al. (2004) Dopaminergic effects of caffeine in the human striatum and thalamus. Neuroreport 15, 281-285.

25. Ebly EM, Schaefer JP, Campbell NR et al. (1998) Folate status, vascular disease and cognition in elderly Canadians. Age Ageing 27, 485-491.

26. Morris SM, Fava M, Jacques PF et al. (2003) Depression and folate status in the US population. Psychother Psychosom 72, 80-87.

27. Brice CF \& Smith AP (2002) Factors associated with caffeine consumption. Int J Food Sci Nutr 53, 55-64.

28. Schwarz B, Bischof H-P \& Kunze M (1994) Coffee, tea and lifestyle. Prev Med 23, 377-384.

29. Minamisawa M, Yoshida S \& Takai N (2004) Determination of biologically active substances in roasted coffees using a diode-array HPLC system. Anal Sci 20, 325-328.

30. dos Santos MD, Almeida MC, Lopes NP et al. (2006) Evaluation of the anti-inflammatory, analgesic and antipyretic activities of the natural polyphenol chlorogenic acid. Biol Pharm Bull 29, 2236-2240.

31. Maes M, Bosmans E, Suy E et al. (1991) Depression-related disturbances in mitogen-induced lymphocyte responses and interleukin-1 beta and soluble interleukin-2 receptor production. Acta Psychiatr Scand 84, 379-386.

32. Maes M, Meltzer HY, Bosmans E et al. (1995) Increased plasma concentrations of interleukin-6, soluble interleukin6 , soluble interleukin-2 and transferrin receptor in major depression. J Affect Disord 34, 301-309.

33. Sarandol A, Sarandol E, Eker SS et al. (2007) Major depressive disorder is accompanied with oxidative stress: short-term antidepressant treatment does not alter oxidative-antioxidative systems. Hum Psychopharmacol 22, 67-73.

34. Scalbert A \& Wiliamson G (2000) Dietary intake of bioavailability of polyphenols. J Nutr 130, 2073-2085.

35. Natella F, Nardini M, Giannetti I et al. (2002) Coffee drinking influences plasma antioxidant capacity in humans. J Agric Food Chem 50, 6211-6216. 